\title{
Field-tunable stochasticity in the magnetization reversal of a cylindrical nanomagnet
}

\author{
Soumik Mukhopadhyay, Amrita Singh, and Arindam Ghosh \\ Department of Physics, Indian Institute of Science, Bangalore 560 012, India
}

\begin{abstract}
The nature of magnetization reversal in an isolated cylindrical nanomagnet has been studied employing time-resolved magnetoresistance measurement. We find that the reversal mode is highly stochastic, occurring either by multimode or single-step switching. Intriguingly, the stochasticity was found to depend on the alignment of the driving magnetic field to the long axis of the nanowires, where predominantly multimode switching gives way to single-step switching behavior as the field direction is rotated from parallel to transverse with respect to the nanowire axis.
\end{abstract}

Traditionally, cylindrical nanomagnets have been of great interest for high density magnetic storage [1], but very recently the dynamics of domain walls (DW) in these systems is also rapidly gaining in importance. A driving factor to this is the absence of "Walker breakdown", with the DWs acting as "massless" particles having zero kinetic energy [2]. However, a generic problem, in both planar or cylindrical nanowires, is the stochasticity associated with the magnetization reversal process. This is manifested in two classes of phenomena: first, the stochasticity associated with diffusive and nondeterministic motion of the DWs in presence of artificial or intrinsic disorder (addressed elsewhere by the same authors [3]); second, the stochasticity related to the nucleation and subsequent propagation of DWs. The latter has been investigated in several planar magnetic films and lithographically patterned nanowires through, for example, size and shape distributions of Barkhausen avalanches, or extraordinary Hall effect etc [4]. Asymmetry in the mode of magnetization switching process on reversal of magnetic field polarity has been investigated using magnetic force microscopy [5], which might as well be related to the stochasticity issue (MFM probes only a fraction of the whole sample). The stochasticity in magnetization reversal in case of cylindrical nanowires, however, is relatively unexplored.

Cylindrical magnets have been the classical template for theoretical study of the mode of magnetization reversal and more importantly the angular variation of the nucleation field, albeit in the limit of an infinite and isotropic cylinder [6]. Many experiments on magnetization reversal in nanoscale magnetic systems have looked at the angular dependence assuming an infinite and isotropic cylinder (or strips), although finite size and anisotropy related effects can be crucial in those cases [15]. Wegrowe et al. could fit their data on angular dependence with the curling mode prediction for an infinite cylinder assuming an activation volume with aspect ratio $2: 1$ (Ref. [16]). Moreover, surface anisotropy or structural defects can also play crucial role and hence cannot be treated within the framework of isotropic magnetization [16]. The principal objective of this article is to explore the effect of angular variation on the stochasticity of magnetization reversal, rather than the variation

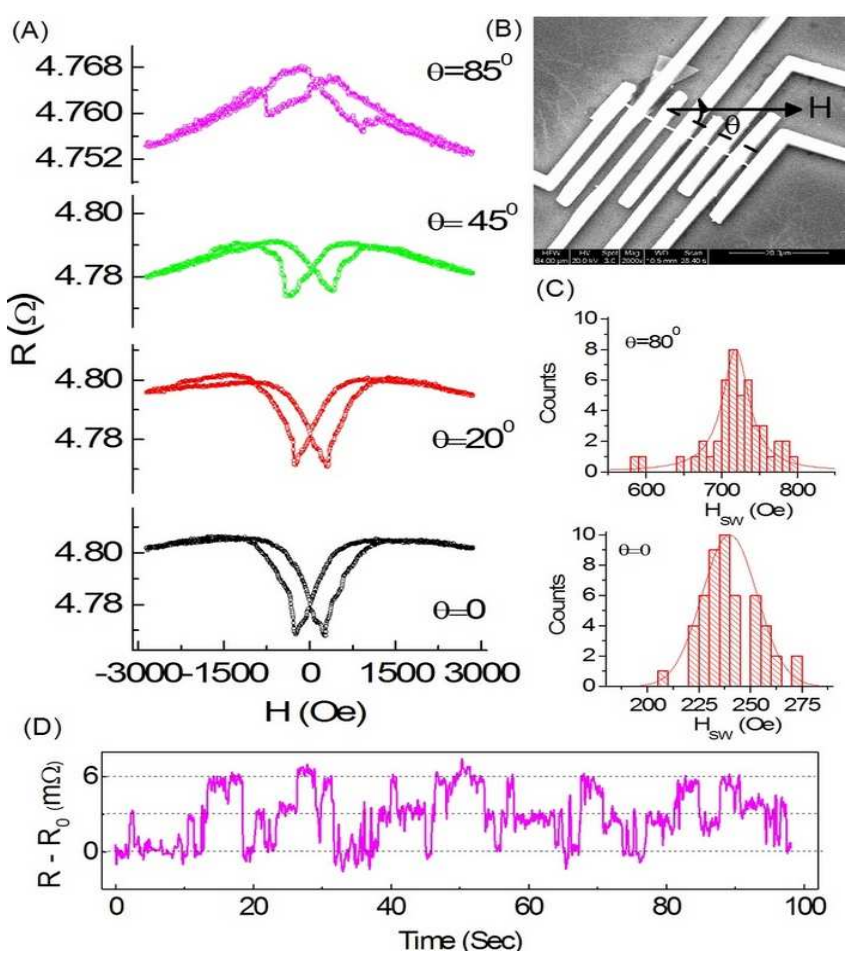

FIG. 1: (A) Magnetic field dependence of resistance at different angles between the applied magnetic field and the nanowire axis suggesting that the angle dependence of $H_{s w}$ has $180^{\circ}$ periodicity. (B) SEM micrograph of a device. (C) The switching field histograms for $\theta=0$ and $\theta=80^{\circ}$. (D) The time dependence of resistance at a static low magnetic field (1.5 Oe) for $\theta=0$ is shown.

in switching field $\left(H_{s w}\right)$, where we show that finite size, disorder, local anisotropy etc. play crucial roles.

Experimentally, we have taken a different approach. Instead of magnetic hysteresis measurement such as using microfabricated SQUID 7] which is limited to low temperature, or magneto-optic Kerr effect (MOKE) [8] which requires significant averaging over many field cycles to improve the signal to noise ratio and hence unsuitable for probing stochastic switching, we have measured time-resolved electrical resistance around $H_{s w}$ to track the nucleation of individual DWs at different angles between the applied magnetic field $(H)$ and the long axis of the cylindrical nanomagnet. Electrical transport in mag- 
netic nano-cylinders have been reported before [9, 16, 17], but primarily to explore the switching mechanism, rather than its stochasticity. Our approach and results may also be relevant to device applications such as DW logic systems [18] or spiral turn sensors [19], which require the use of orthogonal magnetic fields.

Nickel nanowires were electrochemically grown inside anodic alumina templates. The average diameter of the nanowire is $\approx 200 \mathrm{~nm}$, where strong shape anisotropy (aspect ratio $\sim 200$ ) aligns the magnetization along the long axis of the nanowire. Details of the growth process and structural characterization can be found elsewhere [20]. Following growth, nanowires were dispersed on flat silicon oxide substrates, and electron-beam lithography was used to form $\mathrm{Ti} / \mathrm{Au}$ contact pads for electrical measurements on single nanowire as shown in Fig. 1 B. The resistance was measured at room temperature in the four-probe geometry using standard ac lock-in technique.

The magnetoresistance measured at different angles between the applied magnetic field and the current or the long-axis of the cylinder show that the switching field has a minimum at $\theta=0$ and a maximum at $\theta \sim \pm 90^{\circ}$ (Fig. 19A). This $180^{\circ}$ periodicity indicates curling-type magnetization reversal mechanism expected for nanowires of diameter $200 \mathrm{~nm}$ at least when $H$ is parallel to the long axis [6]. The $H_{s w}$ measurement was repeated 50 times. At $\theta=0, H_{s w}$ has a distribution of $12 \%$ around the most probable value of 240 Oe, while at $\theta=80^{\circ}$, the distribution is slightly narrower, about $8 \%$ with a most probable switching field of 720 Oe (Fig. 1C). Although such a narrowing for the transverse direction has been observed before 7] for Nickel nanowires of diameter less than $100 \mathrm{~nm}$ below $6 \mathrm{~K}$, the overall width of $H_{s w}$ distribution is significantly broader in our case, probably due to the larger diameter of the nanowire (which could introduce inhomogeneties thereby lowering the local anisotropy barrier) and enhanced role of thermal activation at room temperature.

For magnetoresistance to be a viable probe to magnetization reversal, it is essential to identify and separate the contributions from anisotropic magnetoresistance (AMR) arising due to spin-orbit scattering, and that from the DWs. To achieve this we have studied the time dependence of nanowire resistance $(R)$ at $H \ll H_{s w}$. The distance between the voltage probes placed midway along the length of the nanowire is $\sim 5 \mu \mathrm{m}$. The nanowire is magnetized in one direction along the long axis and then a small magnetic field is applied in the opposite direction. The time dependence of $\mathrm{R}$ at $H=1.5$ Oe applied parallel to the long-axis of the nanomagnet shows stochastic switching back and forth between discrete multi-level states in $R$ (Fig: 1D). The jumps describe increase in $R$ from its base value $R_{0}(\sim 4.78 \Omega)$ by $\Delta R$ or $2 \Delta R$, where $\Delta R \approx 3 \mathrm{~m} \Omega$ and vice versa. Since $H$ is constant, AMR or Lorentz contributions to $R$ do not change, and hence the observed switching between dif-
A) $\theta=0$
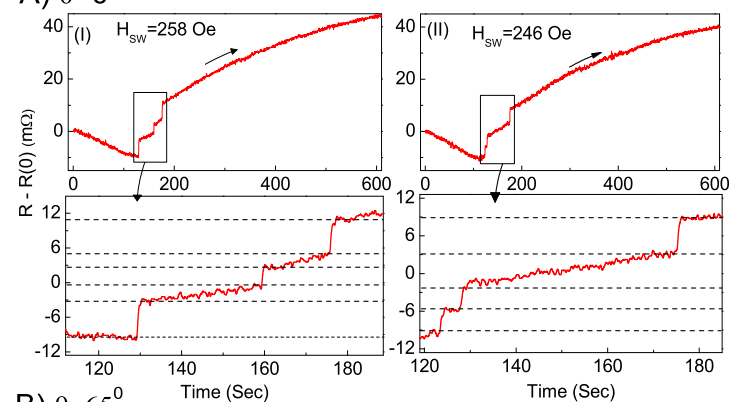

B) $\theta=65^{\circ}$
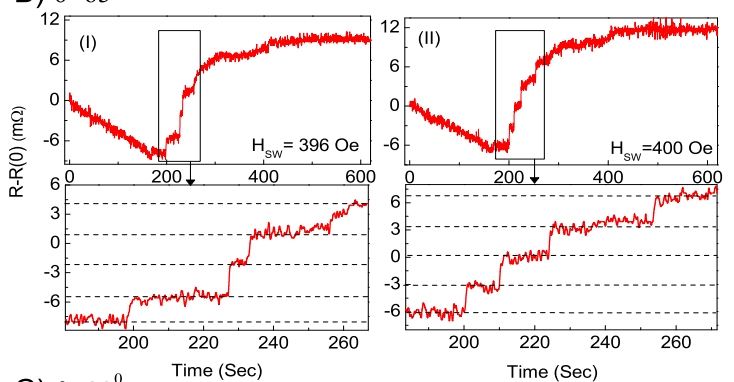

C) $\theta=80^{\circ}$
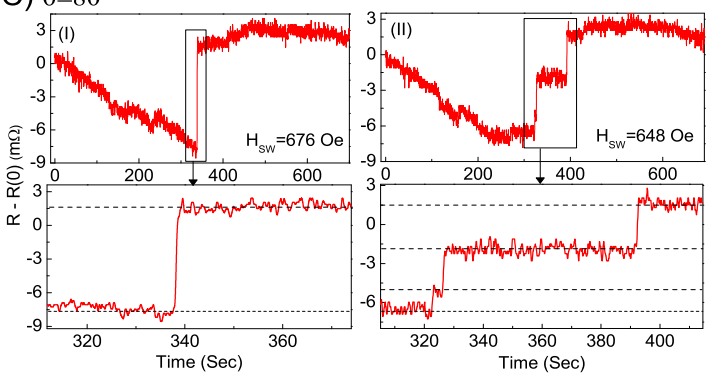

FIG. 2: AI,II) Time dependence of resistance for two measurement cycles with sweeping magnetic field at $\theta=0$ with $R(0) \sim 4.78 \mathrm{~m} \Omega$. The magnetization reversal involves successive stochastic irreversible jumps in resistance which are integer multiples of a $R_{d w}$. Between two jumps, the resistance rises weakly due to AMR. BI,II) Same for $\theta=65^{\circ}$. Resistive jump of size $2 R_{d w}$ is rare at high values of $\theta$. CI, II) Time dependence of resistance showing irreversible single-step and multimode switching at $\theta=80^{\circ}$ for two measurement cycles. Absence of AMR leads to flat resistance plateaus.

ferent $\mathrm{R}$ states can be attributed to the DWs traveling in and out of the region between the voltage probes. Increasing $R$ by $\Delta R$ and $2 \Delta R$ corresponds to the existence of one and two DWs, respectively, between the voltage probes. The positive correction $\Delta R$ can be quantitatively understood from the Levy-Zhang model of spinmixing inside the DWs 21] and has been discussed in detail elsewhere [3].

To study the dynamics of magnetization reversal, $H$ was swept at a rate of $2 \mathrm{Oe} / \mathrm{S}$ across $H_{s w}$ while $R$ was measured as function of time with a resolution of 100 $\mathrm{mS}$. Below $H_{s w}$, as $H$ is increased from zero after be- 
ing saturated in a high $H$ of opposite polarity, the resistance decreases monotonically. Above $H_{s w}$, successive irreversible jumps in the resistance are observed at $\theta=0$ (Fig: 2A). Single-step switching is rare at $\theta=0$, and the striking feature is that the most probable jump size is an integer multiple of $3 \mathrm{~m} \Omega$ (Fig: $3 \mathrm{~A}$ ). The time interval between two successive jumps in a single cycle is nondeterministic and varies from one field cycle to another (Fig. 2A, B). The resistance during that time interval is not constant due to AMR and rises weakly with time (or $H)$. When $H$ is applied along the transverse direction single step switching events become more frequent than multimode switching (Fig: 2 $2 \mathrm{CI}$ ) and the AMR not being significant in transverse direction, $R$ stays constant between successive jumps (Fig: 2 $2 \mathrm{CII}$ ).

The resistance jumps at integral multiple of $\Delta R$ allows us to understand the magnetization reversal mechanism at $\theta=0$. Since $\Delta R$ corresponds to the resistance of a single DW (vortex type since the $\theta$ dependence of $H_{s w}$ suggests curling mode reversal), it can be safely argued that the stochastic multiple jumps in resistance correspond to multiple nucleation events, where jumps of size $6 \mathrm{~m} \Omega$ indicate the nucleation of a pair of DWs. Motion of DWs far below the nucleation field or magnetization reversal due to nucleation of a DW pair is consistent with the time dependent solution of Landau-Lifshitz equation 22] and particularly for transition metal nanowires 23]. The plateaus correspond to DW pinning / small-scale displacement or even rotation of spins (given the weak upward trend in the resistance plateaus at $\theta=0$ due to AMR). Presence of multiple vortex DW suggests that magnetization reversal at $\theta=0$ occurs through localized curling mode with multiple nucleation events. Due to the simplicity of device geometry, it is an open question as to where the DW nucleation starts (possibly at the wire ends or at some local inhomogeneties within the wire) although they are eventually detected within the voltage probes. Similar jumps in the longitudinal resistance have been observed in FePd nanostructure [24]. Multiple vortex walls have also been observed in planar NiFe nanowire of larger width [15].

The stochastic distribution of switching time between each irreversible jump was measured over hundred such switching events. The switching probability was computed from the integral over the switching time histograms for different values of $\theta$ (where multimode switching is predominant), which were fitted with a stretched exponential function $P(t)=\exp -(t / \tau)^{\beta}$, with $\beta$ varying between $1.3-2$ (Fig: $3 \mathrm{~B}$ ). The experimental data for $\theta=0$ follows the overall feature of the theoritical fit although some deviation is observed for low time scale possibly due to small number of switching events in that region. Similar results $(\beta>1)$ have been reported elsewhere, which use more elaborate experimental techniques such as micro-SQUID, magnetic force microscopy or MOKE [25 28]. The probability of not switching as
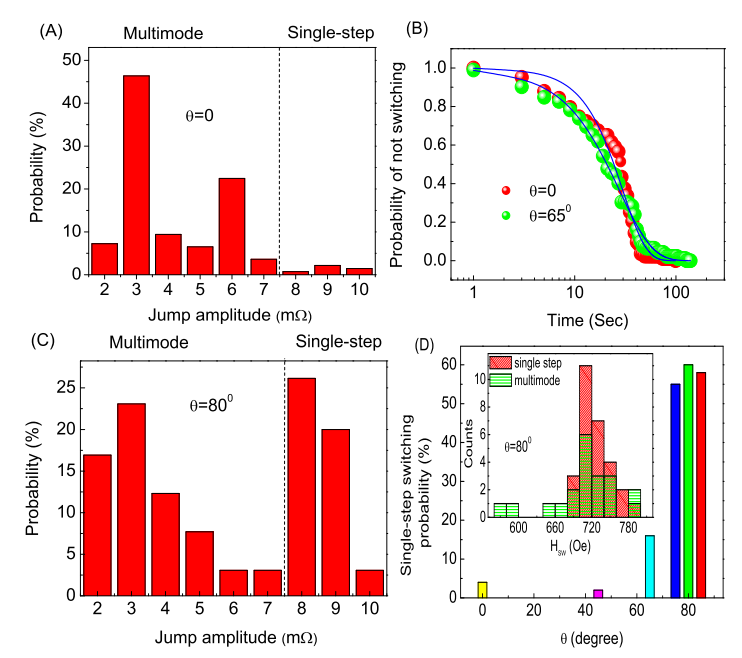

FIG. 3: (A) The jump size distribution during the irreversible switching for (A) $\theta=0$ (B) The enhanced-exponential probability of not switching with $\beta>1\left(\beta=1.86,1.34\right.$ for $\theta=0,65^{\circ}$ respectively) indicates a relaxing multi-valleyed free energy landscape. (C) Jump size distribution for $\theta=80^{\circ}$ (D) Probability of single-step switching as a function of $\theta$. Inset: Switching field distribution for single step and multimode reversal for $\theta=80^{\circ}$ is shown separately.

shown in Fig. 3 B characterized by $\beta>1$ suggests reversal to involve correlated thermally activated processes over a distribution of barrier heights in the free energy landscape, rather than the Neel-Brown picture of thermal activation over a single energy barrier.

The distribution of jump size taken over 50 measurement cycles at different values of $\theta$ shows that for low angles such as $\theta=0$, the most probable jumps are peaked around 3 and $6 \mathrm{~m} \Omega$ while for higher angle such as at $\theta=80^{\circ}$, an additional strong peak appears around $8-9$ $\mathrm{m} \Omega$ with the distribution around $3 \mathrm{~m} \Omega$ being significantly broadened (the resistive jumps of size $6 \mathrm{~m} \Omega$ are rare). It is readily observed that in contrast to the preponderance of multimode switching at lower angle, single-step switching is more frequent at higher angles (Fig: $3 \mathrm{C}, \mathrm{D})$.

The origin of enhanced single-step switching at higher values of $\theta$ remains unclear. Recent micromagnetic simulations have shown that application of transverse $H$ results in the expulsion of the vortex wall leading to magnetization reversal via coherent rotation [9]. However, the experimental evidence is merely based on the deviation of $H_{s w}$ value from that predicted for curling mode at high angle. No such deviation has been observed for $\mathrm{NiFe}$ [13, 15] or Ni nanowires at low temperatures [7]. Wegrowe et al. attributed the deviation from curling mode for cylindrical Nickel nanowire (average diameter $60 \mathrm{~nm}$ ) at room temperature to pinning by surface defects [16]. At higher $\theta$, the value of $H_{s w}$ predicted by 
coherent rotation model is smaller than that from curling model [11]. In our case, the curling mode prediction for a prolate spheroid [29] (assuming an exchange length $d_{e x}=40 \mathrm{~nm}$ and bulk saturation magnetization $M_{S}=0.485 \mathrm{~T}$; the demagnetization factors along the major and minor axes are $D_{z}=0.00015$ and $D_{x}=0.5$, respectively) gives $H_{s w}=140 \mathrm{Oe}$ at $\theta=0$. The underestimation of $H_{s w}$ value at $\theta=0$ has been reported for nanowires with $d / d_{e x}>3$ [30]. The curling model overestimates $H_{s w}$ at $\theta=80^{\circ}$ by a factor of two, while, on the other hand, $H_{s w}$ value predicted by coherent rotation model [31] (where $H_{s w}$ peaks at $\theta=0, \pm 90^{\circ}$ ) is nearly an order of magnitude smaller compared to that observed experimentally at $\theta=80^{\circ}$. Moreover, we have observed that single-step switching on the average involves higher $H_{s w}$ compared to multimode switching (Inset, Fig: 3D). Therefore single step switching cannot be associated with coherent rotation mode. One possible mechanism for single jump reversal is nucleation with very small activation volume and the vortex wall sweeping across the entire length of the nanowire [32]. The question is whether such a scenario is plausible in presence of transverse magnetic field. Transverse $H$ can modify pinning or depinning processes of DWs [33]. Recently, huge enhancement of DW velocity under strong transverse $H$ has been reported [34]. Nonetheless, our experiments prove the stochastic nature of the mode of reversal at high values of $\theta$ with both single-step and multiple-step reversal being possible.

The observation that application of transverse $H$ on a cylindrical nanomagnet has a higher probability to avoid multimode switching could prove to be extremely important for device applications. Large area nucleation pads are used for DW injection into a nanowire where multimode switching due to transverse magnetic field has been a major obstacle [35]. Bryan et al. observed multimode switching in wide planar Permalloy nanowire, due to application of transverse $H$ [8]. Instead of planar wires of rectangular cross-section, nucleation pads with square cross-section (to circumvent lithographic obstacles in fabricating cylindrical nanowire) can be used to reproduce the properties of a cylindrical nanomagnet. Use of softer magnets such as permalloy can reduce the switching field value in the transverse direction.

To conclude, we have employed a simple time dependent magnetoresistance measurement technique to probe the stochasticity of nucleation-mediated magnetization reversal in a cylindrical nanomagnet. When the applied magnetic field is parallel to the long axis of the nanomagnet, the magnetization reversal essentially follows the localized curling mode with more than one nucleation events, whereas when the magnetic field is applied perpendicular to the long axis of the nanomagnet, the mode of reversal is strikingly non-deterministic and can either follow a single-step switching or a multimode switching process.
We acknowledge the Department of Science and technology, Government of India for funding the work. SM thanks CPDF, IISc for financial support.

[1] K. Nielsch, R. B. Wehrspohn, J. Barthel, J. Kirschner, U. Gosele, S. F. Fischer and H. Kronmuller, Appl. Phys. Lett. 79, 1360 (2001).

[2] M. Yan, A. Kakay, S. Gliga, and R. Hertel, Phys. Rev. Lett. 104, 057201 (2010)

[3] A. Singh, S. Mukhopadhyay, and A. Ghosh, Phys. Rev. Lett. 105067206 (2010)

[4] M.-Y. Im, P. Fischer, D.-H. Kim, and S.-C. Shin, Appl. Phys. Lett. 95, 182504 (2009); M.-Y. Im, L. Bocklage, P. Fischer, and G. Meier, Phys. Rev. Lett. 102, 147204 (2009)

[5] S. Y. Suck, V. Neu, U. Wolff, S. Bahr, O. Bourgeois, and D. Givord, Appl. Phys. Lett. 95, 162503 (2009)

[6] A. Aharoni and S. Shtrikman, Phys. Rev. 109, 1522 (1958); H. Frei, S. Shtrikman and D. Treves Phys. Rev. 106, 446 (1957); S. Shtrikman and D. Treves, J. Phys. Radium 20, 286 (1959)

[7] W. Wernsdorfer, B. Doudin, D. Mailly, K. Hasselbach, A. Benoit, J. Meier, J.-Ph. Ansermet, and B. Barbara, Phys. Rev. Lett. 77, 1873 (1996)

[8] M. T. Bryan, D. Atkinson and D. A. Allwood, Appl. Phys. Lett. 88, 032505 (2006)

[9] R. A. Silva, T. S. Machado, G. Gernicchiaro, A. P. Guimaraes, and L. C. Sampiro, Phys. Rev. B 79, 134434 (2009)

[10] A. B. Oliveira, S. M. Rezende, and A. Azevedo, Phys. Rev. B 78, 024423 (2008)

[11] S. Pignard, G. Goglio, A. Radulescu, L. Piraux, S. Dubois, A. Declemy, and J. L. Duvail, J. Appl. Phys. 87, 824 (2000)

[12] R. Lavin, J. C. Denardin, A. P. Espejo, A. Corts, and H. Gmez, J. Appl. Phys. 107, 09B504 (2010)

[13] Y. Rheem, B.-Y. Yoo, B. K. Koo, W. P. Beyerman, and N. V. Myung, J. Phys. D: Appl. Phys. 407267 (2007)

[14] Y. Rheem, B.-Y. Yoo, W. P. Beyermann, and N. V. Myung, Nanotechnology 18, 125204 (2007)

[15] T. Y. Chung, and S. Y. Hsu, J. Appl. Phys. 103, 07 C506 (2008)

[16] J.-E. Wegrowe, D. Kelly, A. Franck, S. E. Gilbert, and J.-Ph. Ansermet, Phys. Rev. Lett. 82, 3681 (1999)

[17] M. Tanase, D. M. Silevitch, C. L. Chien, and D. H. Reich, J. Appl. Phys. 93, 7616 (2003)

[18] D. A. Allwood, G. Xiong, and R. P. Cowburn, J. Appl. Phys. 100, 123908 (2006)

[19] M. Diegel, R. Mattheis, and F. Halder, Sensor Lett. 5, 118 (2007)

[20] A. Singh, D. Chowdhury, and A. Ghosh, Appl. Phys. Lett. 95, 092103 (2009)

[21] P. M. Levy and S. Zhang, Phys. Rev. Lett. 79, 5110 (1997).

[22] J. S. Broz, H. B. Braun, O. Brodbeck, W. Baltensperger, J. S. Helman Phys. Rev. Lett. 65, 787 (1990)

[23] R. Skmoski, H. Zeng, M. Zheng, and D. J. Sellmyer, Phys. Rev. B 62, 3900 (2000)

[24] R. Danneau, P. Warin, J. P. Attane, I. Petej, C. Beigne, C. Fermon, O. Klein, A. Marty, F. Ott, Y. Samson, and 
M. Viret, Phys. Rev. Lett. 88, 157201 (2002)

[25] W. Wernsdorfer, K. Hasselbach, A. Benoit, B. Barbara, B. Doudin, J. Meier, J.-Ph. Ansermet, and D. Mailly, Phys. Rev. B 55, 11552 (1997)

[26] W. Wernsdorfer, K. Hasselbach, A. Sulpice, A. Benoit, J.-E. Wegrowe, L. Thomas, B. Barbara, and D. Mailly, Phys. Rev. B 53, 3341 (1996)

[27] M. Lederman, S. Schultz, and M. Ozaki, Phys. Rev. Lett. 73, 1986 (1994)

[28] D. Atkinson, D. A. Allwood, G. Xiong, M. D. Cooke, C. C. Faulkner, and R. P. Cowburn, Nature Mater. 2, 85 (2003)

[29] A. Aharoni, J. Appl. Phys. 82, 1281 (1997)
[30] R. O'Barr, and S. Schultz, J. Appl. Phys. 81, 5458 (1997)

[31] E. C. Stoner, and E. P. Wohlfarth, IEEE Trans. Magn. 27, 3475 (1991)

[32] R. Hertel, and J. Kirschner, Physica B 343, 206 (2004)

[33] D. Atkinson, D. S. Eastwood, and L. K. Bogart, Appl. Phys. Lett. 92, 022510 (2008)

[34] K. Richter, R. Varga, G. A. Badini-Confalonieri, and M. Vzquez, Appl. Phys. Lett. 96, 182507 (2010)

[35] M. T. Bryan, P. W. Fry, T. Schrefl, M. R. J. Gibbs, D. A. Allwood, M-Y. Im, and P. Fischer, IEEE Trans. Magn. 46, 963 (2010) 